\title{
Severity Four Out of Five
}

National Cancer Institute

\section{Source}

National Cancer Institute. Severity Four Out of Five. NCI Thesaurus. Code C147509.

A severity result of four, on a five level scale. 